\title{
From Margin to Center? Theoretische Aufbrüche in der Geographie seit Kiel 1969
}

\author{
Carolin Schurr ${ }^{1}$ and Peter Weichhart ${ }^{2}$ \\ ${ }^{1}$ Institut für Geographie, Universität Bern, Bern, Schweiz \\ ${ }^{2}$ Institut für Geographie und Regionalforschung, Universität Wien, Wien, Österreich \\ Correspondence: Carolin Schurr (carolin.schurr@giub.unibe.ch)
}

Received: 17 December 2019 - Accepted: 9 March 2020 - Published: 28 April 2020

\begin{abstract}
Kurzfassung. Die Geographie soll sowohl an gesellschaftlicher Relevanz als auch an theoretischer Tiefe gewinnen - so die Forderungen in Kiel 1969. 50 Jahre nach Kiel ist die Bedeutung des Faches unumstritten. Klimastreik, Grenzzäune, Brexit - alle diese aktuellen Ereignisse betreffen Kernthemen der Geographie wie Klimawandel, Migration und Geopolitik.

Aber wer definiert, was gesellschaftlich relevant und damit im Zentrum der Geographie stehen soll? In einem inszenierten Streitgespräch, das als Eröffnungs-Keynote für den Deutschen Kongress in Kiel 2019 entwickelt und hier in leicht veränderter Form abgedruckt wurde, vertreten Carolin Schurr und Peter Weichhart dazu unterschiedliche Positionen: Die Suche nach einem Zentrum des Faches steht dabei der feministisch-postkolonialen Besorgnis über eine solche disziplinäre und disziplinierende Identifikation eines Zentrums gegenüber.

Die Theorieentwicklung des Faches betrachtend, kritisiert Peter Weichhart die zunehmende Zerfaserung und Randständigkeit aktueller Theoriediskussionen. Auf Basis von bell hooks' Buch „From Margin to Center“ zeigt hingegen Carolin Schurr, dass Grundkonzepte der Geographie insbesondere von den gesellschaftlichen und disziplinären Rändern aus hinterfragt und weiterentwickelt wurden. Geographische Zukünfte sehen beide in der Auseinandersetzung mit Fragen der sozialen Gerechtigkeit, wobei das Soziale aus einer posthumanen Dezentrierung heraus gedacht wird.
\end{abstract}

\section{Mythos Kiel 1969}

Peter Weichhart: Ich glaube, wir können in Kiel nicht über die aktuelle Situation der Humangeographie im Jahr 2019 sprechen, ohne zuerst auf Kiel 1969 zurückzublicken.

Carolin Schurr: Da hast Du Recht! Ich assoziiere die studentische Revolution am Deutschen Geographentag in Kiel 1969 mit den gesellschaftspolitischen Aufbrüchen der 1968er-Bewegung in Westdeutschland. Wenn ich an Kiel 1969 denke, dann kommt mir als Erstes das bekannte Bild „Unter den Talaren, Muff von 1000 Jahren“ in den Sinn, das eigentlich von 1967 stammt. Kiel reiht sich für mich in diese Proteste von westdeutschen Studierenden ein, die neben mehr Mitbestimmung und demokratischeren Strukturen an den Universitäten dafür kämpften, die NS-Geschichte aufzuarbeiten.
Im Grundstudium habe ich natürlich davon gehört, welche Umbrüche Kiel für die Geographie gebracht hatte:

1. Vor Kiel war die Geographie scheinbar ,theorie-los“;

2. mit Kiel wurde die klassische Länderkunde bedeutungslos und das Landschaftskonzept infrage gestellt;

3. Kiel hat den Untergang der Einheitsgeographie und die Trennung von Physischer und Humangeographie bewirkt;

4. die Studierenden forderten eine stärkere Orientierung des Faches an Theorie und gesellschaftlicher Relevanz.

Peter, warst Du denn damals in Kiel dabei?

Peter Weichhart: Nun, wirklich „dabei gewesen“ bin ich nicht. Ich war damals Student im 5. Semester 

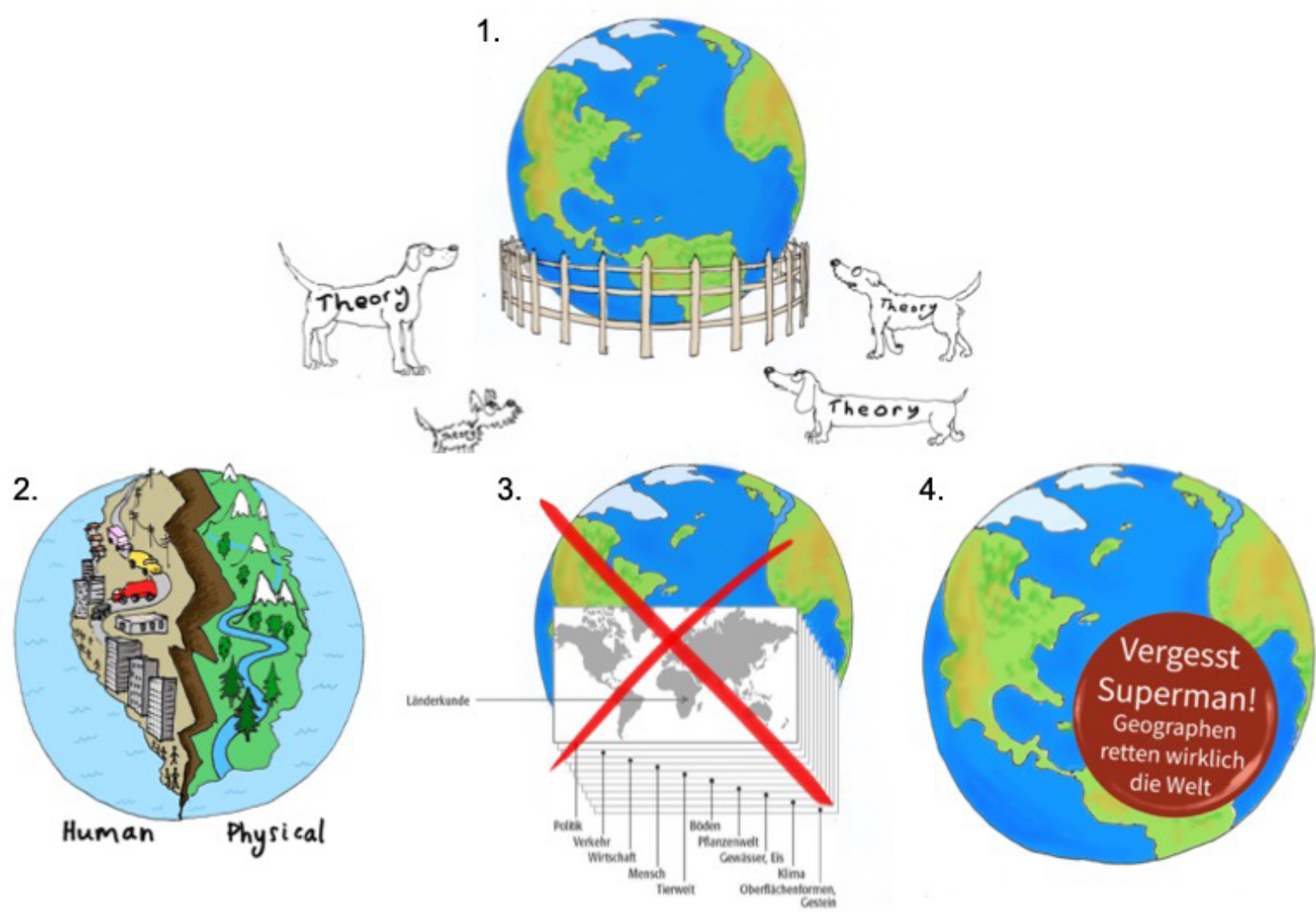

Abb. 1. Umbrüche der Kieler Revolution für die Geographie, (C) Tom Reed Studio.

an der Universität Salzburg. Selbst am Geographentag teilzunehmen wäre für mich schon aus finanziellen Gründen unmöglich gewesen. Aber mein späterer Doktorvater, der Physische Geograph Helmut Riedl, war dabei. Und er hat seinen Studierenden kurz danach in einer seiner Vorlesungen einen ausführlichen Bericht über Kiel geboten.

Seine Schilderung war überaus dramatisch. Kurz zusammengefasst: Die Studierenden und einige revolutionäre Dozierende hätten versucht, die Geographie in ihren Grundfesten zu erschüttern und $\mathrm{zu}$ vernichten. Würden die Forderungen dieser Agitatoren erfüllt, dann wäre der Untergang der Geographie - und damit natürlich auch des Abendlandes - nicht mehr aufzuhalten.

Ich fand das sehr spannend, denn zu dieser Zeit gab es auch in meinem zweiten Fach, der Germanistik, einiges an kritischen Diskussionen. Da ich seit Beginn meines Studiums auch Lehrveranstaltungen in der Philosophie besucht hatte, war mir schon klar, dass es die eine wahre Wissenschaft wohl nicht gibt. Riedls Bericht über Kiel regte mich dazu an, mich näher mit Kiel und den Schriften der Revolutionäre wie Dietrich Bartels (1986) und Gerhard Hard (1970) zu beschäftigen.

Wenn man die von Dir genannten Zuschreibungen mit den fachgeschichtlichen Fakten vergleicht, dann sind wohl einige Korrekturen angebracht:
Die Behauptung, die klassische Geographie sei theoriefrei gewesen, muss zurückgewiesen werden. Wenn man die klassische Geographie metaphorisch mit einer Mehlspeise vergleichen möchte, dann bietet sich das Bild eines Krapfens (norddeutsch: Berliner) an.

Eine runde Sache, klar strukturiert und abgrenzbar, mit einem eindeutigen Zentrum. Dieser süsse Kern, der die klassische Geographie ausmachte, war ein elaboriertes Theoriesystem, das, im Zentrum des Faches stehend, seine Einzelteile verknüpfte und damit auch die „Einheitsgeographie“ begründete. Es bestand aus zwei Elementen, dem klassischen Landschaftskonzept und dem länderkundlichen Schema. Das Landschaftskonzept wurde durch die sogenannte „Integrationsstufenlehre“ abgesichert. Das länderkundliche Schema war keineswegs eine blosse „Kochbuchanweisung“ für das Schreiben von Länderkunden, sondern eine ausgewachsene inhaltliche Theorie. Die gleichsam „logische“ oder „naturentsprechende“ Aufeinanderfolge der Kapitel einer Länderkunde würde nämlich den kausalen Verursachungszusammenhängen von der physischen Struktur hin zu den kulturellen Gegebenheiten entsprechen. Das ist die zentrale theoretische Behauptung des länderkundlichen Schemas.

Carolin Schurr: Ganz schön geodeterministisch. Die Natur bestimmt die Lebensweise der Menschen. Mit 


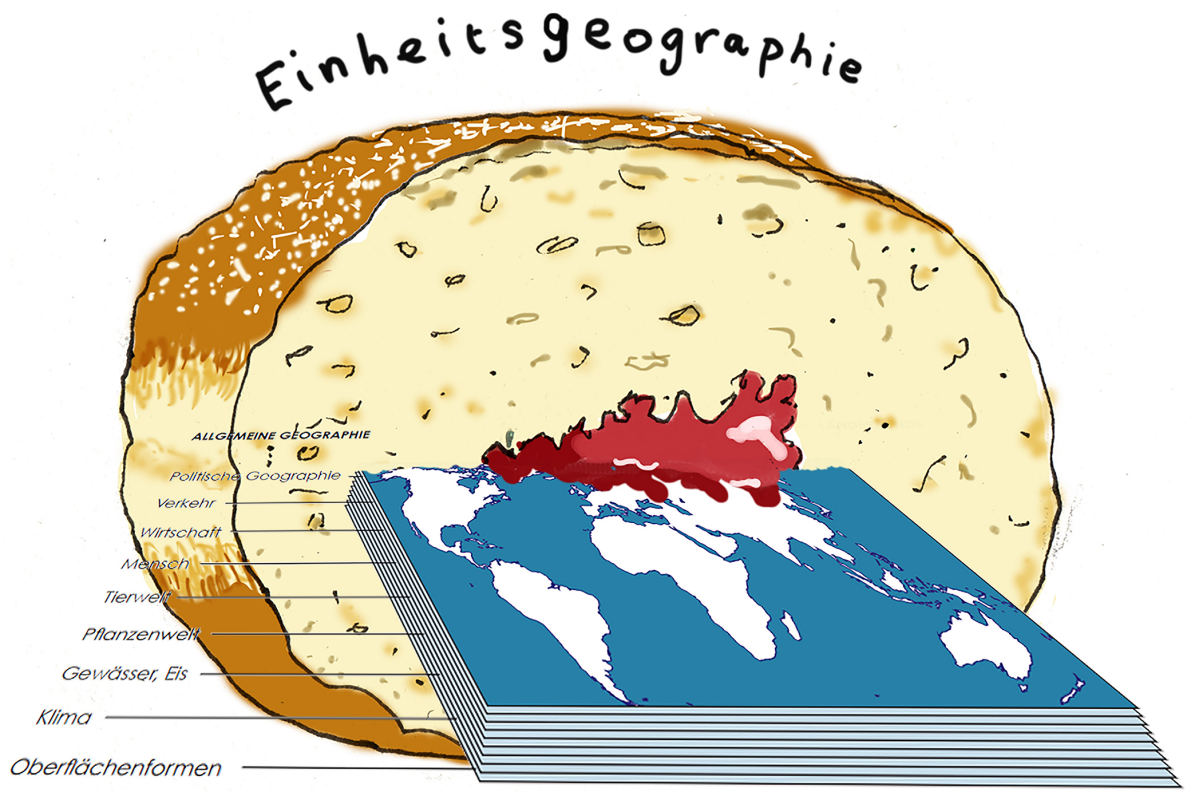

Abb. 2. Der Berliner der Einheitsgeographie, (C) Tom Reed Studio.

Benno Werlen (2014:296) kann man sagen, dass sich hier der problematische Kern der traditionellen Geographie zeigt: „die naturalisierende Reduktion des Sozial-Kulturellen“. Hat Kiel Deiner Meinung nach denn erfolgreich das Landschaftskonzept aus der Geographie verbannt und die Länderkunde abgeschafft?

Peter Weichhart: Das würde ich in dieser Schärfe nicht sagen. Das klassische Landschaftskonzept hat bis heute als Residualparadigma überlebt, wie die Tagung „Aktuelle Perspektiven und Herausforderungen für Landschaftsforschung, -planung und -entwicklung" in Kiel im September 2019 (im Vorfeld des DKG) zeigt. Auch die Länderkunde heute in der Regel als „Regionale Geographie“ bezeichnet - wird weiterhin hochgehalten. Peter Sedlacek (1979:7) konstatierte zehn Jahre nach Kiel, dass ,niemals zuvor in der Geschichte der deutschen Geographie ähnlich viele Länderkunden geschrieben und veröffentlicht [wurden] wie in den vergangenen zehn Jahren" - allen Legitimationsproblemen zum Trotz. Axel Borsdorf (2019) hat unlängst unter dem Titel „Länderkunde abgeschafft? Zur Bedeutung der Regionalgeographie im deutschen Sprachraum vor und 50 Jahre nach Kiel" ein leidenschaftliches Plädoyer für die Länderkunde vorgelegt. Der Text endet mit der Aussage: „Wir erkennen an, ... dass die Regionale Geographie dabei ist, ihre Bedeutung zu festigen. Es scheint so, als ob Kiel überwunden ist" (Borsdorf, 2019:358).

Carolin Schurr: Wenn man die gegenwärtige Publikationspraxis betrachtet, dann scheinen Landschafts- und Länderkunden allerdings nur eine recht marginale Position im Gesamtgefüge des Faches einzunehmen. Werfen wir noch einen Blick auf die dritte Forderung von Kiel: die Trennung der Physischen und Humangeographie.
Peter Weichhart: Diese ist meiner Ansicht nach in der Zwischenzeit allerdings so gut wie vollständig vollzogen, wie die Zitationsanalysen von Steinbrink et al. (2014) belegen: Zwischen Physischer und Humangeographie finden kaum inhaltliche Fachgespräche statt, sie zitieren einander kaum noch.

Um beim Bild der Backwaren zu bleiben: Die Zitationsanalysen der deutschsprachigen Geographie ähneln einer Brezel (oder bairisch: Brezn), in der die drei Teile Physische Geographie, Humangeographie und Geographiedidaktik - recht hermetisch voneinander abgeschirmt sind.

Die in den letzten Jahren erkennbaren Bemühungen, die drei Teilbereiche der Geographie in Form einer „Re-Integration“ in eine neue Einheitsgeographie wieder zusammenzuführen, sind allesamt gescheitert. Das liegt am Fehlen einer für beide Bereiche verbindlichen gemeinsamen theoretischen Grundlage. Die von mir vorgeschlagene „Dritte Säule“ (vgl. z.B. Weichhart, 2003), die neue Interaktionstheorien aus dem Bereich der Sozialökologie nutzt und damit eine Neukonzeption zur Erforschung von Gesellschaft-Umwelt-Interaktionen entwickelt, wurde nur vereinzelt aufgegriffen (vgl. z.B. Gebhardt, 2003; Schneider, 2006).

Die Forderung nach einer Trennung von Physio- und Humangeographie war übrigens schon lange vor Kiel virulent. Diesbezüglich war Kiel also keineswegs eine Revolution, sondern nur der Höhepunkt einer längst erkennbaren Entwicklung.

Carolin Schurr: Was war dann wirklich neu an Kiel?

Peter Weichhart: Neu war erstens, dass die schon vorher schwelenden Konflikte nicht im Hinterzimmer und unter vorgehaltener Hand, sondern in einer öffentlichen Grossver- 


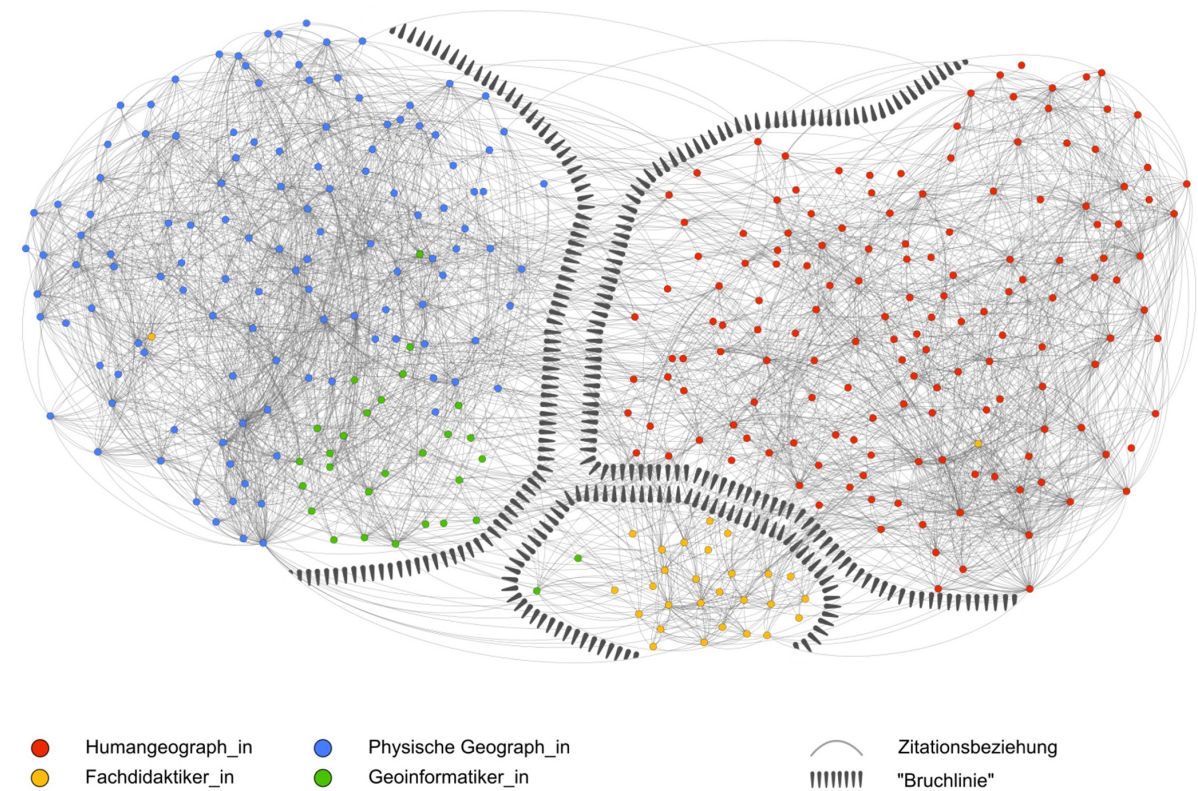

Abb. 3. Zitationsanalyse der deutschen Geographie (Steinbrink et al., 2014).

anstaltung klar und pointiert ausgesprochen wurden. Neu war auch, dass sich die Studierenden dabei mit überzeugenden Argumenten eingebracht haben.

Neu war vor allem aber, dass mit Kiel - sehr stark angestossen durch die Studierenden - ausdrücklich die Forderung nach einer Theorieorientierung des Faches erhoben und eine höhere gesellschaftliche Relevanz eingefordert wurde.

Dabei könnte man drei Stossrichtungen unterscheiden.

Erstens, eine eher pragmatisch berufsorientierte Ausrichtung interpretierte ,gesellschaftliche Relevanz“ vor allem in Hinblick auf die Raumplanung und Berufsfelder ausserhalb der Schule.

Die zweite Forderungslinie orientierte sich an einer raumwissenschaftlichen Position, die der kritisch-rationalen Perspektive von Dietrich Bartels (1986) entsprach. Die Geographie solle auf der Grundlage der Philosophy of Science zu einer quantitativ ausgerichteten Disziplin umgebaut werden, die nach Raumgesetzlichkeiten sucht und mit nomologischen Theorien operiert. Diese Forderung wurde von manchen Fachvertreter_innen als ein Hauptverdienst der Revolution von Kiel interpretiert. Dabei wurde allerdings übersehen, dass die deutschsprachige Geographie hier (Plessner, 1959 [1935]) als eine ,verspätete Disziplin“ angesehen werden muss, denn die quantitative Revolution, die bei uns mit Kiel 1969 erst begann, war im anglophonen Sprachraum längst vollzogen.

In einem Artikel, der 1967 publiziert wurde, konstatierte Leslie Curry (1967), dass die quantitative Revolution längst abgeschlossen sei. Zwar finden sich in den zwei Jahrzehnten nach Kiel zahlreiche deutschsprachige Veröffentlichungen, die formalisierte Raummodelle und sophistizierte Methoden der analytischen Statistik verwenden. In der Zwischenzeit sind rein quantitative Ansätze aber nur mehr selten zu finden. Carolin Schurr: Ich finde es beachtlich, dass 50 Jahre nach Kiel zumindest in der Humangeographie so wenig von der „quantitativen Revolution“ übrig geblieben ist.

Peter Weichhart: Die dritte Stossrichtung der studentischen Forderung nach Theorien hat unter, gesellschaftlicher Relevanz" etwas völlig anderes verstanden. Man orientierte sich analog zur 1968er-Bewegung an der Kritischen Gesellschaftstheorie der Frankfurter Schule und an Karl Marx. Das entscheidende Kriterium der Theorie ist hier die Fähigkeit, Kritik an den herrschenden Verhältnissen zu üben. Damit wurde für die Geographie das Tor zur Rezeption gesellschaftstheoretischer Ansätze weit aufgestossen. Und das halte ich für die eigentlich zentrale Leistung der Revolution von Kiel.

Carolin Schurr: Und wie ist die Geschichte dann weitergegangen?

\section{Theorieentwicklung seit Kiel 1969}

Peter Weichhart: Aus meiner Sicht gab es dann in der deutschsprachigen Geographie eine mehr als zehn Jahre dauernde Schrecksekunde. Nur sehr zögerlich wurden bei uns die Anregungen der „Behavioral Geography“ aufgegriffen, die im englischen Sprachraum ebenfalls mit dem Anspruch auf gesellschaftliche Relevanz vertreten wurde. Die gesellschaftliche Relevanz des Faches könne erhöht werden - so die Behavioral Geographers -, würde man nur den Ballast der quantitativen Ansätze und Modelle über Bord werfen. Und dann ging es Schlag auf Schlag weiter. 


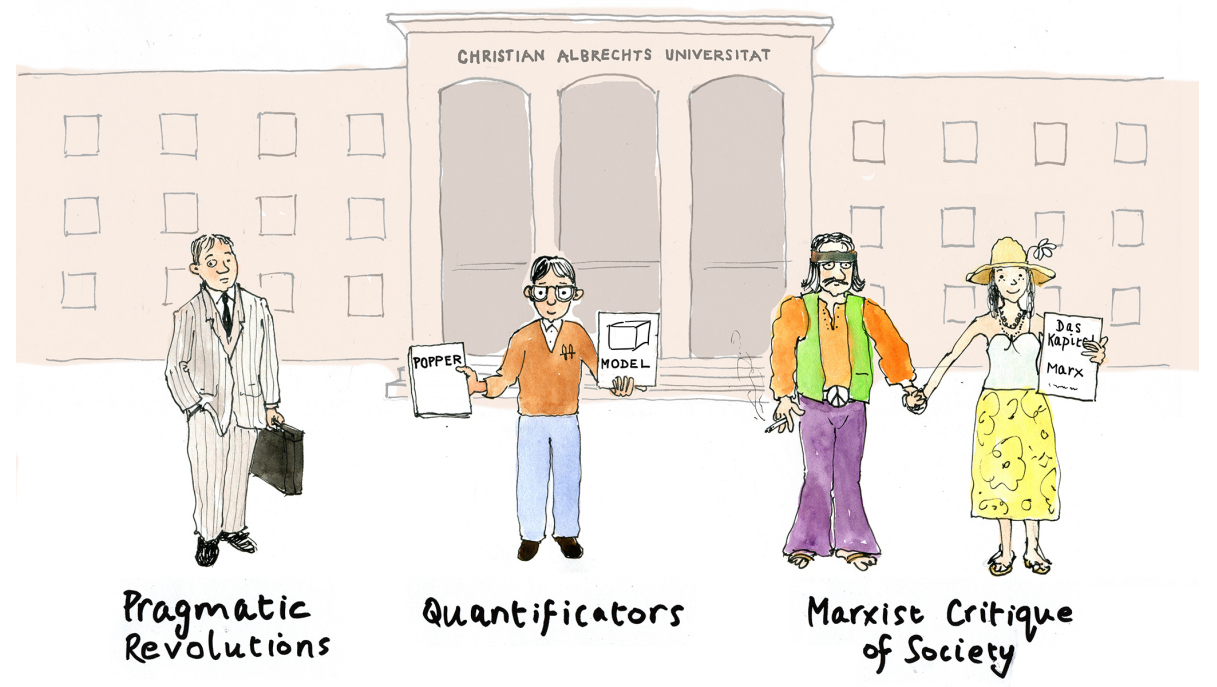

Abb. 4. Die Revolutionär_innen von Kiel 1969, (C) Tom Reed Studio.

Carolin Schurr: Ja, ab den späten 1980er-Jahren wurde die Öffnung des Faches gegenüber disziplinübergreifenden Sozialtheorien immer deutlicher erkennbar: Humanistische Geographie, Marxistische Geographie, Benno Werlens Handlungstheorie, feministische sowie schliesslich poststrukturalistische und postkoloniale Theorien fanden Einzug in die Geographie.

Peter Weichhart: Mit allen diesen Theorien haben wir das, was ich ein „Multiparadigmenspiel“ nenne (Weichhart, 1987), denn es gibt keine rational begründbare Möglichkeit, einen dieser Ansätze als den „besseren“ oder „richtigen“ Weg zu qualifizieren. Die Humangeographie ist ziemlich unübersichtlich geworden, und ich persönlich stehe dieser Situation eigentlich ziemlich hilflos gegenüber.

\section{Was/Wer is(s)t im Zentrum?}

Peter Weichhart: Das vorher wirkmächtige Zentrum, der konzeptionelle Kern der Geographie, hat sich aufgelöst, und ich frage mich, was im Zentrum der Geographie steht. Der „Krapfen“ wurde zum Donut, mit einem „Loch“ als Mitte.

Mein aktueller Eindruck von unserem Fach ist, dass wir uns auf „einer grossen Spielwiese“ befinden, ,auf der sich zahlreiche wunderschöne Steckenpferdchen tummeln, deren Bewegungen aber weitgehend unkoordiniert und ohne gemeinsame Choreographie erfolgen. Jedes dieser prächtigen Steckenpferde ist an spannenden Einzelthemen und zweifellos sehr wichtigen Fragen orientiert. Man verliert bei der Betrachtung aber ein wenig den Überblick und sucht etwas ratlos nach verbindenden Gemeinsamkeiten oder einem roten Faden, mit dessen Hilfe das Zentrum der Bewegungen gefunden werden könnte“ (Weichhart, 2019 [2008]:410).

Carolin Schurr: Aber das passt doch zu der Vielfalt und Ausdifferenzierung alltäglichen Lebens seit der „Postmoderne“! Für mich ist die ,grosse Spielwiese“ ein Zeichen dafür, dass die Geographie so vielfältig, chaotisch und unübersichtlich geworden ist wie die Welt, die sie zu beschreiben versucht (Law, 2004). Wenn so die Geographie aussieht, dann finde ich das einen sehr positiven Befund. Denn für mich liegt genau in der Undiszipliniertheit der Geographie - hier im doppelten Sinne von nicht von Autoritäten zensiert und nicht in die engen Grenzen einer wissenschaftlichen Disziplin gezwängt - ihr Mehrwert. Was stört Dich an der „grossen Spielwiese“ mit den vielen ,wunderschönen Steckenpferdchen"'?

Peter Weichhart: Manchen der aktuellen sozialgeographischen Themenfelder sind (aus meiner Sicht) eine gewisse Subtilität, Randständigkeit oder gar eine esoterische Note gemeinsam. Sie sind zwar zweifellos innovativ und erörtern Fragestellungen, die bislang nicht oder unzulänglich behandelt wurden: Geography of friendship, Atmosphärenforschung, Psychoanalytic Geography. Aber sie sind in der Regel meilenweit entfernt von den aktuellen gesellschaftlichen Problemfeldern, als da sind: Klimawandel, wachsende Fremdenfeindlichkeit, neuer Nationalismus, Aufstieg rechtsradikaler politischer Kräfte, weiteres Aufgehen der Schere zwischen Arm und Reich. Carolin Schurr: Als ich diese Kritik an aktuellen Arbeiten das erste Mal in Deinem Lehrbuch "Sozialgeographie“ (Weichhart, 2019 [2008]) gelesen habe, kam mir sofort die Geschichte der Feministischen Geographie in den Sinn. Sie galt lange Zeit für maskuline GeographEN als 


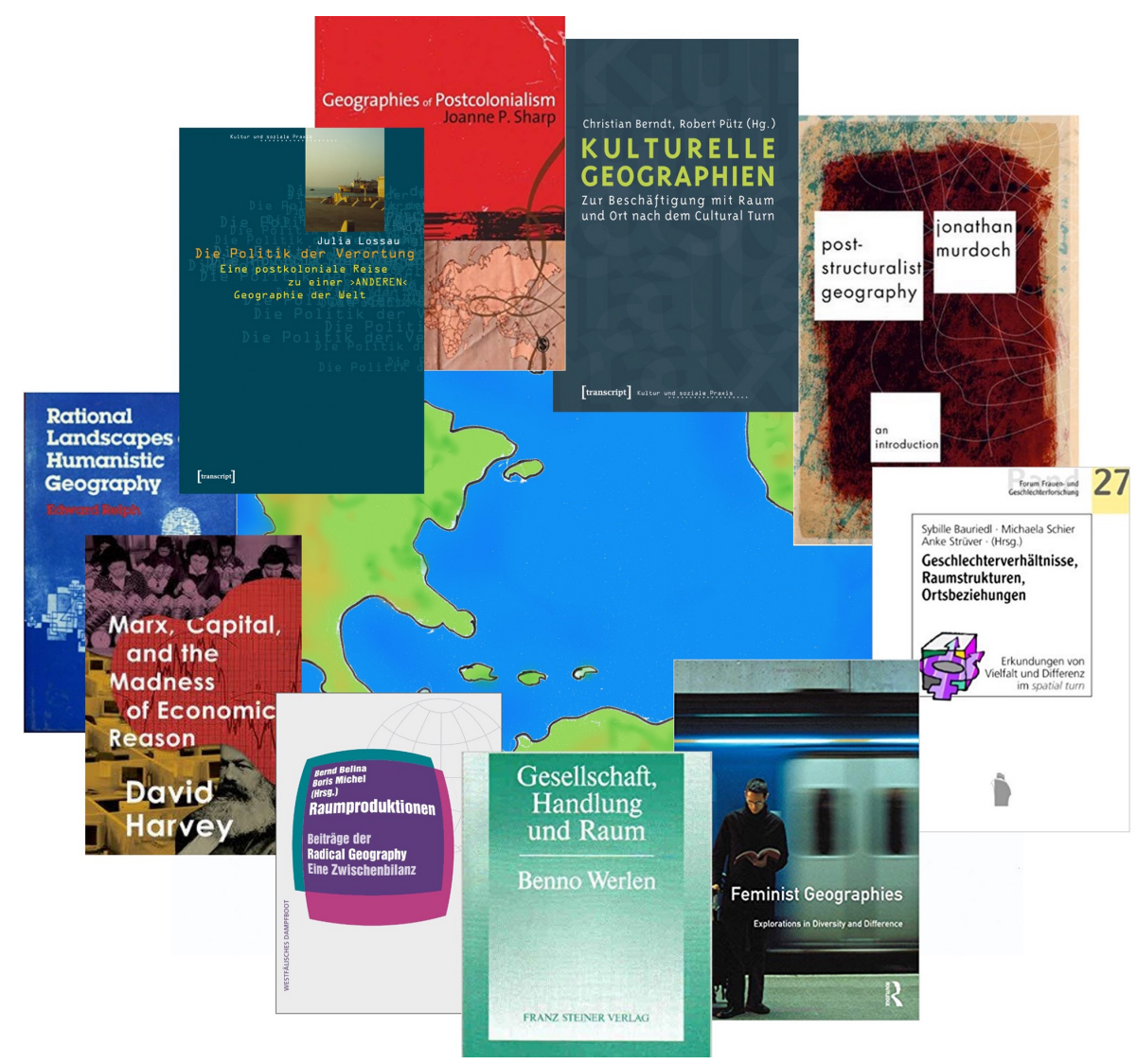

Abb. 5. Multiparadigmenspiel der Geographie.

„randständig“. In ihrem Paper „On not excluding half of the human in human geography" zeigten Janice Monk und Susan Hanson im Jahr 1982, dass geographische Forschungsfragen für die Lebenswirklichkeiten von Frauen und Männern gleichermassen relevant sind, aber meist nur die Erfahrungen der Männer analysiert wurden, wenn es zum Beispiel um Pendlerwege im Zuge der Suburbanisierung ging. Indem feministische Geograph_innen Fragen der sozialen Differenz als relevante Analysekategorie einführten, ermöglichte feministische Forschung ein differenzierteres Welt-Bild.

Peter Weichhart: Ja, Fragen der Differenz sind sicher zentral geworden für die Geographie. Aber ich bezweifle, dass Fragen der Differenz allein ein neues Zentrum für die Geographie schaffen können.

Carolin Schurr: Zumindest haben Geographien der Differenz ein zentrales Anliegen: Fragen der Gerechtigkeit ins Zentrum geographischer Forschung zu rücken.

Aber mit Deiner Suche nach einem neuen Zentrum für die Geographie stehst Du nicht allein. In der Stadtgeographie fordern Neil Brenner und Christian Schmid (2015) eine neue epistemologische Fundierung, und Michael Storper und Allen Scott (2016) suchen nach einer gemeinsamen Sprache, die der Zersplitterung des Feldes der Stadtgeographie

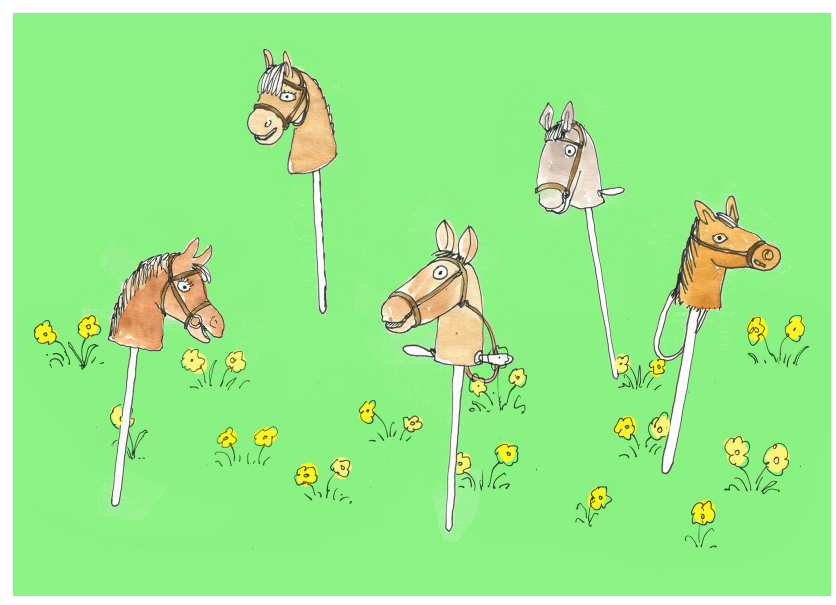

Abb. 6. Die grosse Spielwiese der Geographie mit ihren Steckenpferdchen, (C) Tom Reed Studio.

entgegenwirkt. Auch in der Wirtschaftsgeographie wurde in den letzten Jahren von Jamie Peck und Chris Olds (Peck, 2012; Peck und Olds, 2007) die Frage nach dem „Kern“ dieser Subdisziplin mithilfe des Bilds des Donuts erörtert. Peck und Olds zeigen, wie die Wirt- 


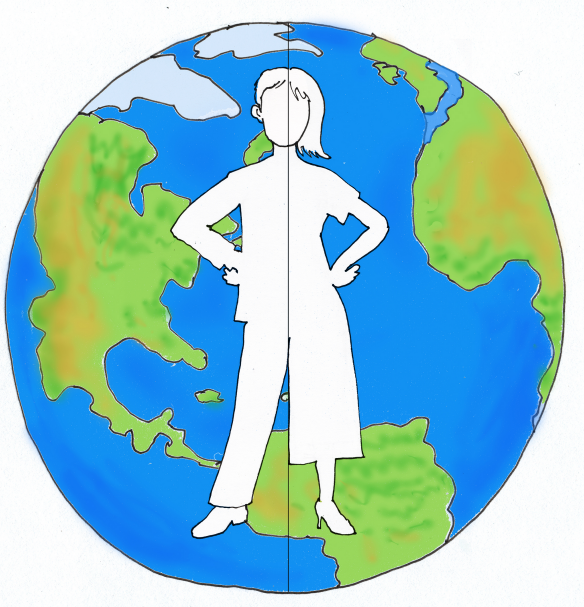

Abb. 7. On not excluding half of the human in human geography, (C) Tom Reed Studio.

schaftsgeographie ursprünglich ein extrem zentralisiertes Feld war, in dessen Zentrum als Forschungsobjekt die Wirtschaft der Industrienationen des Globalen Nordens stand, die aus polit-ökonomischer Perspektive analysiert wurde. Im Zuge des „Cultural Turns“ hat sich das Feld radikal geöffnet. Wirtschaftsgeograph_innen sind mit unterschiedlichen theoretischen Ansätzen in Dialog getreten, die von Politischer Ökologie über Cultural Studies bis hin zu feministischen und postkolonialen Theorien reichen (Barnes, 2001; Berndt und Boeckler, 2008; Bonds, 2013; Castree, 2004; Gibson-Graham, 2014; Jessop und Oosterlynck, 2008; McDowell und Court, 1994; Oberhauser, 2000; Pickles, 2012; Pollard et al., 2009; Thrift und Olds, 1996). Der Kern des Faches wurde Donut-gleich ausgehöhlt, und die Kräfte an den Rändern wurden buchstäblich zentrifugal und immer durchlässiger zu Nachbar- und anderen Subdisziplinen.

Peter Weichhart: Ja, eine ähnliche Entwicklung kann man wohl für die Humangeographie als Ganzes attestieren. Es ist einfach furchtbar unübersichtlich geworden.

Carolin Schurr: Aus feministischer und postkolonialer Sicht finde ich diese zentrifugale Öffnung hin zu einer postdisziplinären Welt sehr positiv. Denn jede Form, ein Zentrum oder einen Kanon zu etablieren, sind Formen eines disziplinären Imperialismus, der zwangsläufig zu Ausschlüssen von bestimmten Forschungsgegenständen, theoretischen Zugängen und unterschiedlichen sprachlichen und kulturellen Wissenschaftsgemeinschaften führt.

Die Mode von Handbüchern ist ein gutes Beispiel für den Versuch, einen Kanon im Hinblick auf Themen und Theorien in der Geographie festzuschreiben.

Peter Weichhart: Nehmen wir etwa das „Sage Handbook of Geographical Knowledge" von Agnew und Livingstone (2011:2), das für sich beansprucht, sich kritisch mit den Konzepten auseinanderzusetzen, die im Zentrum der Geographie stehen. Sie machen immerhin den Versuch

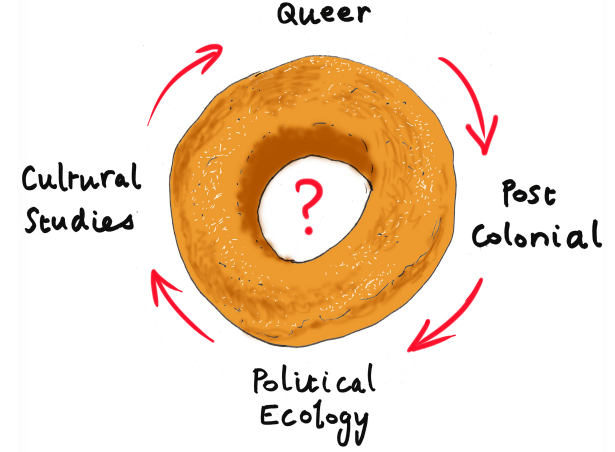

Abb. 8. Die zentrifugalen Kräfte, die den Donut Geographie aushöhlen, () Tom Reed Studio.

in ihrem Handbuch, so etwas wie den Kern der Disziplin auszumachen. Was stört dich daran?

Carolin Schurr: Den Kern der Geographie und dessen „reworking" definieren dabei 48 Autor_innen, alle 48 aus Anglo-Amerika, davon nur 7 Frauen (Schurr et al., 2020). Wenn man im Sinne von Donna Haraway (1988) Wissen - oder hier geographical knowledge - immer als „situiert“ versteht und ernst nimmt, dass die Positionalität der Autor_innnen massgeblich die Perspektiven des Textes beeinflusst, dann finde ich einen solchen Versuch, den Kern der Disziplin festzulegen, höchst problematisch. Das Beispiel des Sage Handbooks zeigt, dass die Etablierung eines Zentrums oder Festschreibung eines Kanons eine sehr maskuline und koloniale Geste ist, die ihr „Territorium“ versucht $\mathrm{zu}$ verteidigen. Diese imperiale Geopolitik des Wissens (Mignolo, 2000, 2001; Müller, 2019; Quijano, 2000), die durch ein solches Handbook produziert wird, definiert klar, welches Wissen als hegemonial gilt und im Zentrum der disziplinären Debatten steht und welches Wissen als irrelevant an den Rand gedrängt wird.

Peter Weichhart: Ja, verteidigen klingt nach kolonialem Abstecken von Ländereien. Aber trotzdem: Es geht doch hierbei auch um die Zukunft unserer Disziplin.

Carolin Schurr: Ich finde die Pluralisierung des Faches von den Rändern sehr wichtig und richtig. Für mich geht die Aushöhlung der Mitte mit der Diversifizierung der Disziplin selbst einher: einerseits im Hinblick auf die Subjekte, die sich selbst Geograph_in nennen; andererseits im Hinblick auf die Forschungsthemen, die wir bearbeiten, die immer stärker verschiedene Gruppen und Anliegen in den Fokus rücken, die in der Gesellschaft keine hegemoniale Position (Globaler Norden, weiss, männlich, heterosexuell) einnehmen. Ausserdem kann man anhand der Feministischen Geographie und den Black Geographies gut zeigen, dass theoretische Innovationen oft von den Rändern der Disziplin bzw. der Gesellschaft ausgehen.

Peter Weichhart: Hast Du für eine solche theoretische Innovation von den Rändern ein Beispiel? 


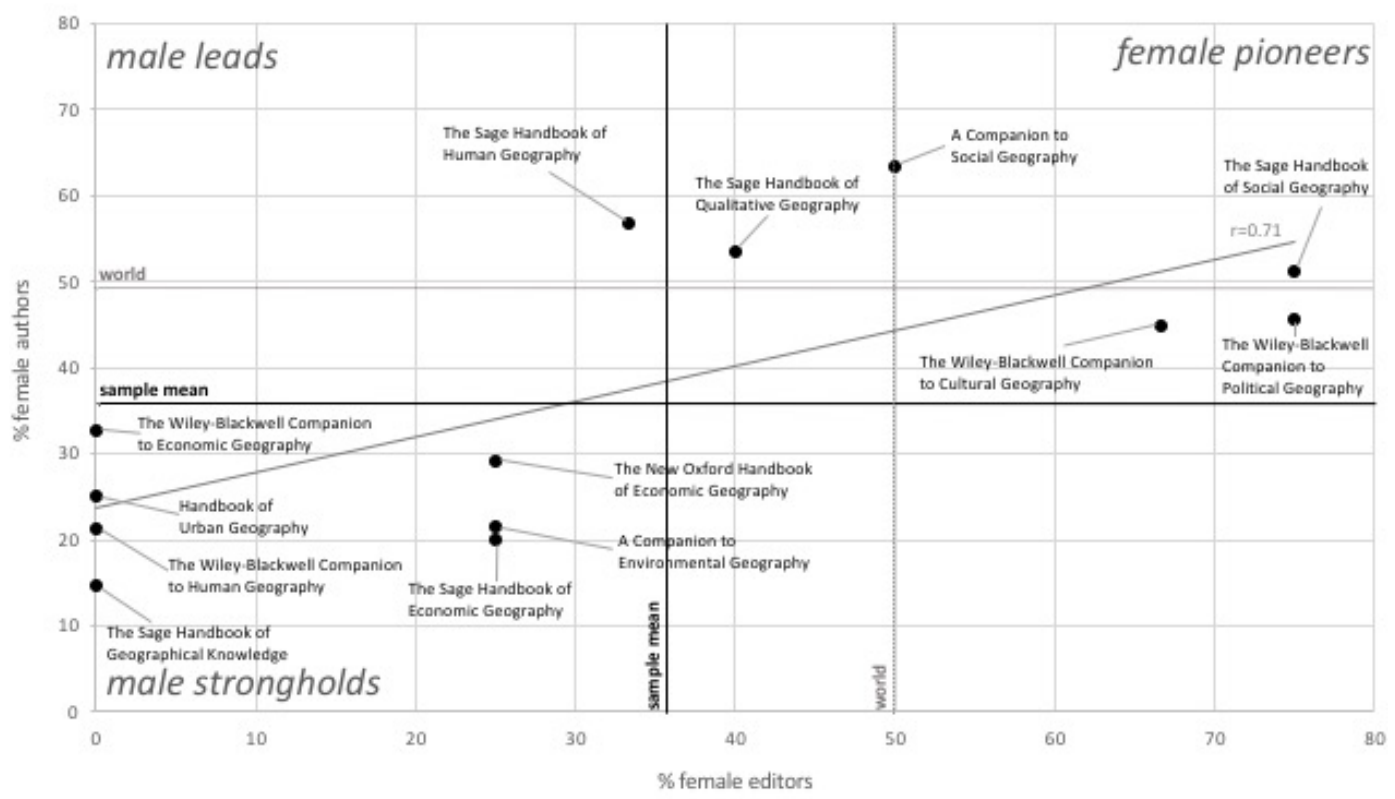

Abb. 9. „Gendered gatekeepers“ in Handbüchern der Geographie (Schurr et al., 2020).
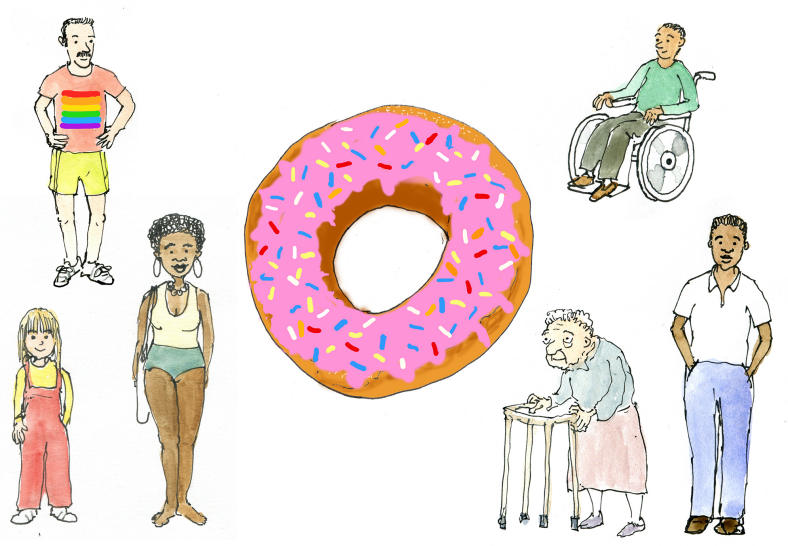

Abb. 10. Die Pluralisierung des Donuts Geographie, (c) Tom Reed Studio.

\section{Theoriebildung aus den Rändern}

Carolin Schurr: Nehmen wir dazu das Konzept von „Rand“, Marginalität selbst als Beispiel. Das Konzept der Marginalität oder Peripherie spielt in geographischen Modellen und Theorien ganz unterschiedlicher Schulen eine zentrale Rolle - man könnte es als ein Grundkonzept der Geographie verstehen.

Seit den 1990er-Jahren haben Poststrukturalistische, Queer-Feministische, Postkoloniale Geographien und Black Geographies Fragen um Zentralität und Marginalität neu gedacht. Ausgangspunkt dazu war dabei für viele bell hooks' Artikel mit dem Titel „Choosing the Margin as a Space

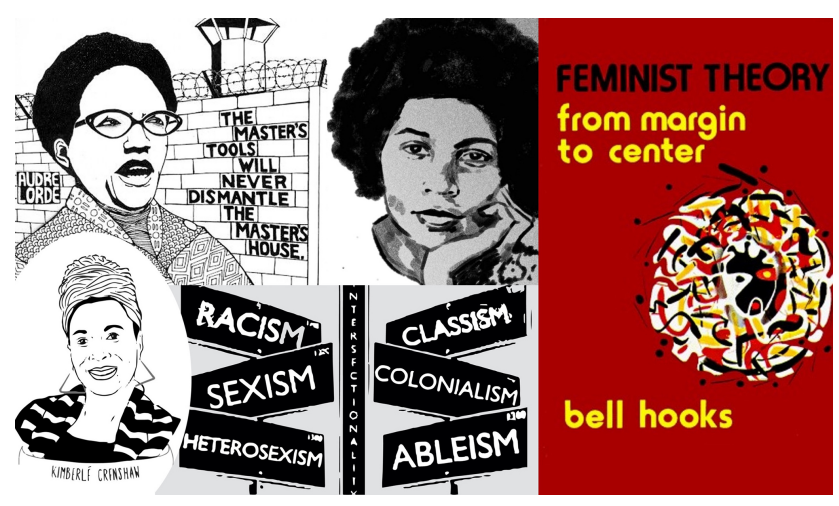

Abb. 11. Audre Lorde, Kimberley Crenshaw und bell hooks als Vordenkerinnen für Geographien des Widerstands.

of Radical Openness“ (Hooks, 1989), den sie in ihrem Buch „Feminist Theory: From Margin to Center“ (Hookes, 2000) weiterentwickelte. Tatsächlich kommen viele der in der Geographie aktuell verwendeten Konzepte, um Marginalisierungen zu beschreiben, aus schwarzem feministischen Gedankengut: Kimberle Crenshaws (1989) „Kreuzung“ von verschiedenen Diskriminierungserfahrungen, auf dem das mittlerweile in der Geographie weit verbreitete Konzept der Intersektionalität beruht (Brown, 2012; Hopkins, 2017; Valentine, 2007), oder Audre Lordes (1984) „The Master's Tools Will Never Dismantle the Master's House“, das nicht mit den Werkzeugen des Masters niedergerissen werden kann (Koopman, 2008; Mollett und Faria, 2018), sind räumliche Metaphern für Marginalität.

Peter Weichhart: Was genau versteht hooks unter „Margin“? 


\section{Carolin Schurr:}

To be in the margin is to be part of the whole but outside the main body. As black Americans living in a small Kentucky town, the railroad tracks were a daily reminder of our marginality. Across those tracks were paved streets, stores we could not enter, restaurants we could not eat in, and people we could not look directly in the face. Across those tracks was a world we could work in as maids, as prostitutes, as long as it was in a service capacity. We could enter that world but we could not live there. We had always to return to the margin, to cross the tracks, to shacks and abandoned houses on the edge of town (Hooks, 1989:20).

Was ich mit bell hooks' Beschreibung des Randes gerne zeigen möchte ist, dass die konkrete gelebte Erfahrung von Marginalität zu theoretischer Innovation geführt hat, indem Räumlichkeit neu gedacht wurde als subjektiv erlebtes und immer auch verkörpertes Phänomen. Wie Anke Strüver (2010, 2014) es so passend ausgedrückt hat: Der Körper fungiert hier als Scharnier zwischen Subjekt und Gesellschaft, zwischen physisch-materieller Umwelt und diskursivem Raum. Aus ihrer eigenen gelebten Erfahrung heraus, dass ihre weiblichen, Schwarzen Körper am Rand der Gesellschaft stehen, haben (Schwarze) Geograph_innen den Körper als relevanten Raum und Massstabsebene in die Geographie eingeführt (Colls und Evans, 2013; Longhurst, 2001; Longhurst und Johnston, 2014; McDowell, 2009; Nelson, 1999). Im deutschsprachigen Raum kritisierten Bauriedl et al. (2000:130), dass „,der Körper im geographischen Alltag bisher kaum thematisiert [wurde].“

Peter Weichhart: Ja, es ist wunderlich. Die Humangeographie bezieht sich auf den Menschen, der ohne seinen Körper nicht ist, und trotzdem hat sie ihn so lange aussen vor gelassen.

Carolin Schurr: Die Integration des Körpers als geographischer Raum und weitere Skalenebene jenseits des Lokalen war jedoch zentraler Ausgangspunkt für wichtige theoretische Innovationen im Fach in den folgenden Jahren: Performativitäts-, Praktiken- und Affekttheorien - sie alle rückten den Körper ins Zentrum des geographischen Forschungsinteresses und bauten dabei unter anderem auf feministisch-geographischen Konzeptualisierungen des Körpers auf - oft ohne diese Genealogie zu würdigen.

Peter Weichhart: Du meinst, in der „Mainstream-Humangeographie“ ist mehr feministische Theorie versteckt, als wir dies auf den ersten Blick sehen?

Carolin Schurr: Ja, genau. Die Hinwendung zum Alltäglichen, zu Praktiken, zu Emotionen, zum Körper - dies sind alles Fragen, die im Zentrum feministischer Theoriebildung stehen. Mittlerweile werden sie von Geograph_innen für sich beansprucht und wurden zum Beispiel im Rahmen von Nigel Thrifts (2008) Non-representational Geography oder Theodore Schatzkis (2002) Praktikentheorie in den anglophonen und deutschen geographischen Mainstream gebracht.

Peter Weichhart: Und was macht ihr als feministische Geograph_innen damit?

Carolin Schurr: Im Rahmen des DFG-Netzwerks „Feministische Geographien des New Materialism“ sind wir diesen feministischen Genealogien nachgegangen. Wir haben uns damit beschäftigt, wie jüngere Arbeiten zum New Materialism auf den Arbeiten feministischer Marxist_innen oder der feministischen Wissenschafts- und Technikforschung aufbauen (Ahmed, 2008; Haraway, 1988).

\section{Dezentrierung des Menschen}

Carolin Schurr: Diese Arbeiten aus dem Bereich des Feministischen Materialismus und Posthumanismus, welche die Handlungsmacht des Menschen radikal dezentrieren, haben meiner Ansicht nach sehr viel Potenzial für die Geographie. 50 Jahre nach Kiel bieten diese Theorien vielleicht eine Möglichkeit, Physische Geographie, Humangeographie und Geographiedidaktik wieder in Konversation zu bringen. Denn in einer Zeit, in der die Umwelt regelrecht unter unsere Haut geht - durch genveränderte Lebensmittel, Mikroplastik oder Giftstoffe aus Industrie und Landwirtschaft, ist ein solcher intradisziplinärer Dialog nötiger denn je.

Peter Weichhart: Was fordern denn diese beiden Perspektiven des Feministischen Materialismus?

Carolin Schurr: Die Perspektive der Feminist Materialisms betont die Notwendigkeit einer kritischen Erweiterung des Cultural Turns unter Rückgriff auf die lange Tradition von feministischer Wissens- und Technikforschung. Sie unterstreicht, dass menschliche Körper und Subjekte nicht nur durch gesellschaftliche Interaktionen, Strukturen und Diskurse, individuelle Wahrnehmungen und Praktiken konstituiert werden, sondern auch durch Intra-Aktionen und „Verschränkungen“ zwischen Biologischem und Sozialem (Alaimo und Hekman, 2008; Barad, 2008; Marquardt und Strüver, 2018; Schurr, 2017; Strüver, 2019).

Peter Weichhart: Da wären wir wieder bei meiner dritten Säule. Seit Kiel 1969 und der Trennung von Physischer und Humangeographie mussten wir erkennen, dass sich die Grenzen zwischen Natur und Kultur aufgelöst haben. Die Welt ist mit hybriden Phänomenen - oder Verschränkungen des Physischen und Sozialen - bevölkert. Denn ja, Gentechnologie und Klimawandel sind Beispiele dafür, dass das auf der Natur-Kultur-Dichotomie aufgebaute ontologische Modell spätestens seit Donna Haraway (1991) und Bruno Latour (1993) obsolet geworden ist.

Carolin Schurr: Ja, die Erkenntnis, dass Natur und Kultur nur relational gedacht werden können, ist alt. Und doch denke ich, dass Posthumanistische Geographien, die durch Arbeiten der Feminist Materialisms inspiriert sind, noch einen Schritt weitergehen. Sie verstehen den Menschen selbst immer schon als mehr-als-menschlich oder als 


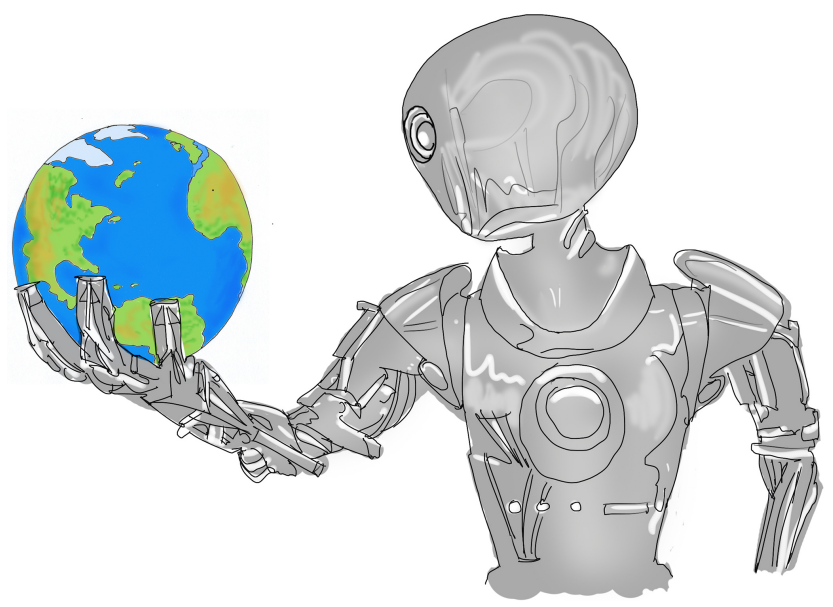

Abb. 12. Die Cyborgisierung des Menschen und des Planeten, (C) Tom Reed Studio.

transkorporeal, wie Stacy Alaimo (2010) es nennt. Egal, ob man von den Interdependenzen zwischen unterschiedlichen Spezies ausgeht (Haraway, 2008; Hodgetts und Lorimer, 2015), vom Mensch als Cyborg Assemblage, dessen Grenzen durch neue Formen der Technologie, Robotik und künstlicher Intelligenz hybrider werden (Black, 2014; Franklin, 2006; Haraway, 1991; Latour, 2002) oder von der Wirkmächtigkeit von Materie selbst (Braun, 2011; Müller und Schurr, 2016; Tolia-Kelly, 2013), die Plastizität des menschlichen Körpers entsteht durch Intra-Aktionen mit anderen Menschen, Technologien, Tieren, Ökologien und Umwelten.

Peter Weichhart: Soweit stimme ich dir zu. Aber was bedeutet das für das Unterfangen, die Mitte des Donuts „Geographie“" neu zu füllen?

Carolin Schurr: Posthumane Geographien dezentrieren die Handlungsmacht des Menschen radikal, er ist nicht mehr der allein Handelnde und Agierende, sondern agiert nur innerhalb von sozio-technischen und sozio-ökologischen Agencements. Wir Menschen könnten von diesen hybriden Gefügen aus gemeinsam mit Tieren, Umwelt, Technologie und Materie Widerstand gegen den gegenwärtigen kapitalistischen Anthropozentrismus leisten, der momentan das Gesamtsystem Erde zerstört. Ganz im Sinne von bell hooks' „Margin as a Space of Resistance“ als einen Ort des Widerstands, der Möglichkeiten, der Hoffnung und der Freiheit (Giudice und Giubilaro, 2015; McKittrick, 2011; Nelson, 2006) könnten wir als Geograph_innen empirisch fundierte Utopien entwickeln, wie so eine mehr-als-menschliche Ordnung aussehen könnte.

Peter Weichhart: Das klingt sehr poetisch, Ränder als Orte des Widerstands zu sehen, und mir gefällt als Geograph die Möglichkeit, von dort aus alternative, neue Welten zu denken. Für mich besteht das Ziel geographischer Forschung durchaus im Sinne von hooks darin, dazu beizutragen, neue Welten zu schaffen, indem Fragen sozialer Gerechtigkeit

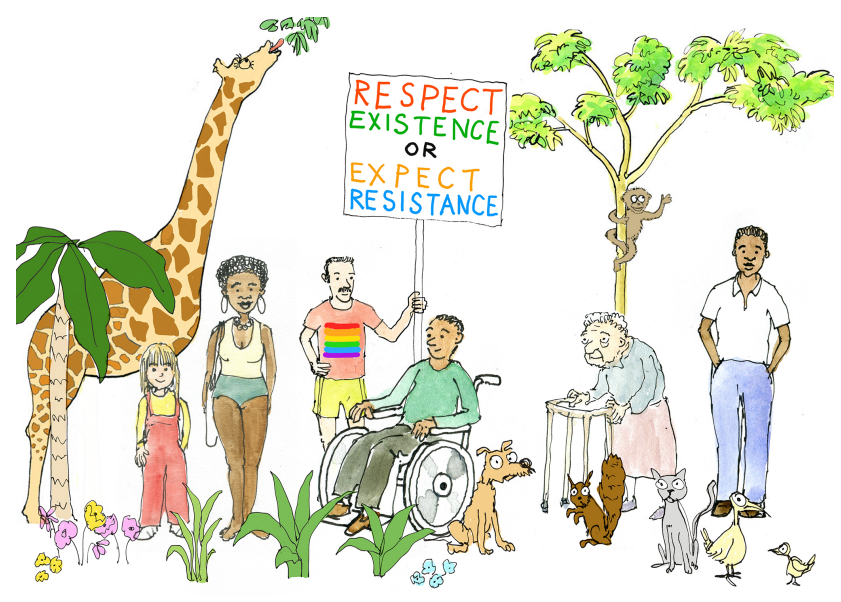

Abb. 13. Mehr-als-humaner Widerstand gegen die Zerstörung des Planeten Erde, (C) Tom Reed Studio.

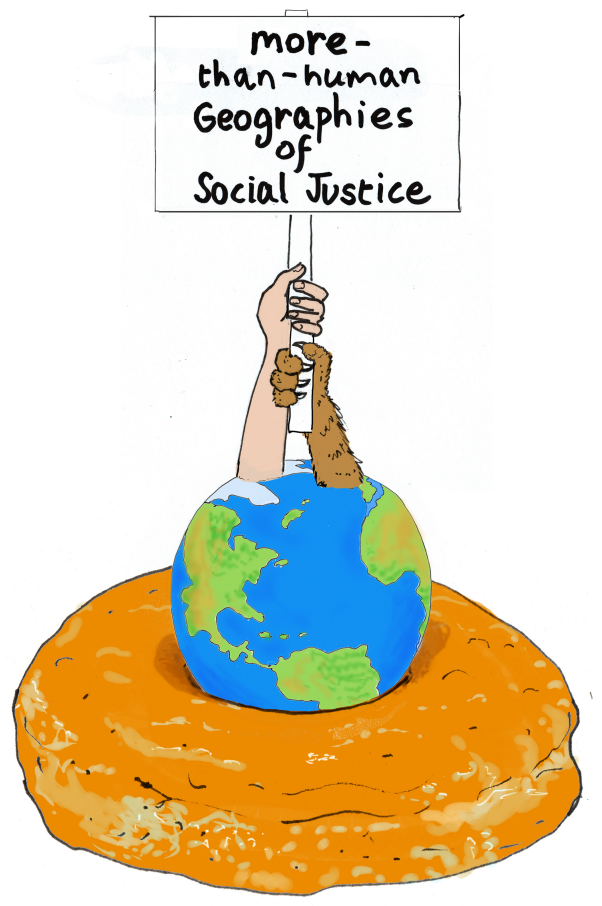

Abb. 14. Mehr-als-humane Geographien der Gerechtigkeit, (c) Tom Reed Studio.

wieder ins Zentrum der Forschung gerückt werden. Man könnte diese inhaltliche Dimension als Universal-Ethik bezeichnen. Für mich sind solche Geographien der Gerechtigkeit eine Möglichkeit, das Donut-Loch zu füllen, um die vielen Steckenpferdchen zusammenzuführen und zu koordinieren.

Carolin Schurr: Dabei sollten im Sinne meines Plädoyers für Posthumane Geographien Fragen der sozialen Gerechtigkeit nicht mehr nur vom Menschen aus gedacht werden. Ich würde deshalb hier eher von mehr-als-humanen Geographien 
der Gerechtigkeit sprechen. Wichtig finde ich, dass wir als Geograph_innen dabei nicht nur Formen der Dominanz, Unterdrückung und Ausbeutung (von bestimmten Menschen, Tieren, Ökosystemen, Technologien) kartieren, sondern $\mathrm{zu}$ einer gerechteren und inklusiveren (im Sinne von mehr-als-humanen) Konzeptualisierung dieser Grundkonzepte hinarbeiten.

\section{Zentrierende Suchbewegungen...}

Peter Weichhart: Eine solche Auseinandersetzung mit den Grundkonzepten der Geographie passt gut zu meinem zweiten Vorschlag, wie das Donut-Loch gefüllt werden kann. Ich persönlich bin der Meinung, dass wir bei unseren Überlegungen jetzt eine Position erreicht haben, die es mir erlaubt, Mutmassungen über sinnvolle Antworten auf die Frage nach dem Zentrum, der Mitte, des Faches Geographie anzustellen. Ich stimme mit Dir überein, dass solche Antworten keinesfalls dazu führen dürfen, dass neue Orthodoxien entstehen. Ein derartiger Versuch liesse sich aus einem Abstraktionsprozess ableiten, durch den grundlegende geographische Konzepte und Begriffe sowohl konkret-räumlich als auch metaphorisch gedeutet werden können.

Carolin Schurr: Das klingt sehr abstrakt. Was genau stellst Du Dir da vor?

Peter Weichhart: Unsere Überlegungen zu Zentrum und Rand können hier gut als Beispiel dienen. Diese beiden Begriffe können auf unterschiedlichste Weise interpretiert werden. Die Relation Zentrum - Rand könnte man als Fundamentalie bezeichnen. Im Sinne der Mathematik (Fischer, 2006) verstehe ich unter Fundamentalien Invarianten, die sich als Basisbegriffe in allen Paradigmen unseres Faches und in seiner gesamten Ideengeschichte finden lassen. Es gibt ein Basiswissen in der Geographie, ,das auf Fundamentalien beruht, die ihrerseits durch physikalische und kognitive Universalien im Zusammenhang mit der Lebensbewältigung des Menschen bestimmt sind“ (Fischer, 2006:163). Durch die Struktur unseres kognitiven Apparates bedingt, handelt es sich gleichsam um anthropologische Konstanten, die zumindest über gewisse Zeiten hin universell wirksam sind. Carolin Schurr: Du meinst, solche Fundamentalien würden für verschiedene Paradigmen, ja sogar Felder der Geographie - also für Human - und Physische Geographie gleichermassen funktionieren?

Peter Weichhart: Für mich ist hier sehr wichtig, dass es keine Rolle spielt, welche paradigmenspezifische Brille man dabei aufsetzt. Es handelt sich eben deshalb um eine Fundamentalie, weil dieses Konzept sowohl in der Quantitativen, der Feministischen, der Marxistischen, der Humanistischen, der Handlungstheoretischen Geographie oder in der Regionalen Geographie Sinn ergibt und mit Nutzen verwendet werden kann. Ich behaupte, dass es eine ganze Reihe solcher Fundamentalien gibt, die im

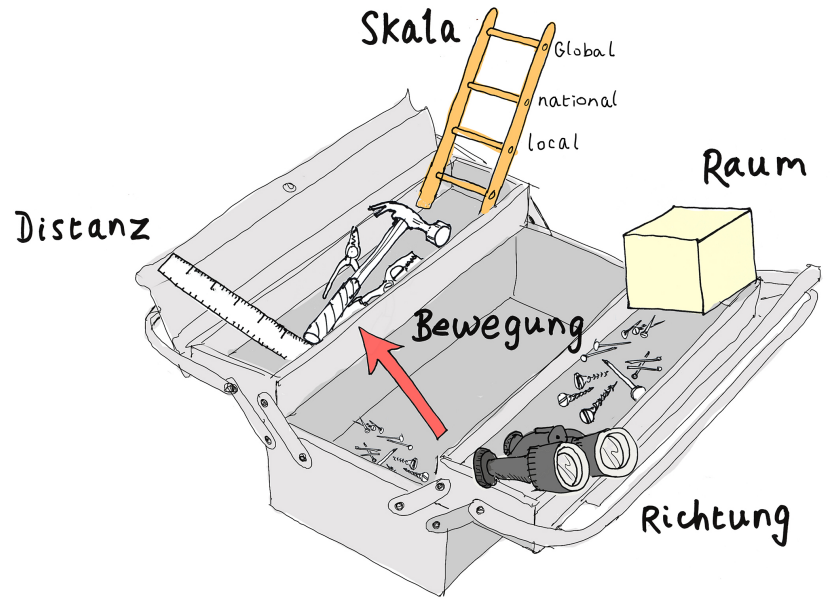

Abb. 15. Der Werkzeugkoffer der Geographie, (C) Tom Reed Studio.

Zentrum des Faches stehen. Zu den Fundamentalien zählen etwa die Konzepte Räumlichkeit (spatiality), Ort (place), Mobilität, Skalen, Kontextualität, Struktur, Prozess, Agency, Differenz, Disparitäten oder Grenze. Ich würde dazu auch die Grundkonzepte des Spatial Approach zählen, nämlich Distanz, Richtung und Konnektivität. Das Gleiche gilt für die „Fundamental Conditions“ (vgl. die Zusammenstellung bei Kramer, 2012:85), die Torsten Hägerstrand als Ausgangspunkt seiner Zeitgeographie formuliert hat. Auch Agencements/Assemblages, Netzwerk, Rhizom oder das Konzept der sozialen Figurationen im Sinne von Norbert Elias (1978) könnten zu den Fundamentalien gezählt werden.

Die Gesamtheit solcher Fundamentalien sehe ich als eine Art Instrumentarium oder Werkzeugkoffer an, mit dessen Hilfe wir vielfältige Problemlagen der Geographie sinnvoll behandeln können. Ich habe keine Ahnung, wie gross das Universum der geographischen Fundamentalien ist. Aber als Geograph_innen sollten wir darüber systematisch nachdenken.

Carolin Schurr: Der Begriff der Fundamentalie gefällt mir zwar nicht so gut, weil er ähnlich wie Deine Suchbewegung nach einem Zentrum der Geographie etwas „Fundamentalistisches“ hat. Aber das Bild des Werkzeugkoffers, in dem sich Werkzeuge ganz unterschiedlicher Art finden und dessen Zusammensetzung sich immer wieder ändert, wenn alte Werkzeuge nutzlos werden oder verloren gehen oder neue entwickelt werden und hinzukommen, gefällt mir gut. Für mich eröffnen diese unterschiedlichen Werkzeuge geographische Perspektiven auf ganz unterschiedliche Phänomene. Wichtig ist dabei für mich, dass der Werkzeugkoffer immer geöffnet bleibt und damit die Geographie offen für neue Perspektiven ist.

Peter Weichhart: Die Fundamentalien sind als abstrakte Perspektiven zu sehen, die von jedem Paradigma oder Ansatz aus in Wert gesetzt werden können. Ein ,disziplinärer 
Imperialismus“ kann von ihnen also nicht ausgehen. Mir ist es abschliessend wichtig zu betonen, dass ich es sinnvoll und vernünftig finde, für das Fach eine neue Mitte zu schaffen und damit das ,Donut-Loch“ zu füllen.

Carolin Schurr: Warum hältst Du einen Kern oder ein Zentrum des Faches eigentlich für so wichtig?

Peter Weichhart: Weil ich immer wieder sehe, dass die gegenwärtige Forschungspraxis derart fragmentiert ist, dass zwischen den verschiedenen „Denkinseln“ kaum mehr Kommunikation besteht. Diese Zerfaserung halte ich auch aus fachpolitischer Sicht für bedenklich. Durch die eingeschränkte Binnenkommunikation wird auch die Kommunikation nach aussen und gegenüber den Studierenden erschwert. Auf die Frage, wofür die Geographie eigentlich steht, gibt es so viele divergierende Antworten, dass wir selber unsicher werden. In zahlreichen Gesprächen mit Kolleg_innen und auf mehreren Tagungen habe ich immer wieder gesehen, dass es innerfachlich eine Art Sehnsucht nach einer neuen Mitte gibt.

Carolin Schurr: Aus der Sicht einer Feministischen und Postkolonialen Geographie kann ich eine solche Sehnsucht nicht erkennen. Vielleicht findet sich hier doch der erhoffte Generationenkonflikt wieder. Auch in der angelsächsischen Debatte sind es meist die männlichen, weissen Geographen einer älteren Generation, die ein neues Zentrum, eine neue gemeinsame Sprache oder eine neue Epistemologie für die Geographie fordern. Gegen solche neuen universalen Theorien wie zum Beispiel die der ,planetary urbanization“ (Brenner und Schmid, 2011) leisten hingegen vor allem Frauen, People of Color sowie Wissenschaftler_innen aus dem Globalen Süden und Osten Widerstand (McLean, 2018; Oswin, 2018, 2019; Peake et al., 2018; Reddy, 2018; Ruddick et al., 2018). Sie plädieren dafür, dass es sicher nicht immer einfach ist, mit der Ungewissheit einer fehlenden Mitte umzugehen. Aber das Aushalten dessen, was Du „multiparadigmatische Struktur“ genannt hast, ist notwendig, um die Disziplin jeder Form der hegemonialen Disziplinierung zu entziehen.

Peter Weichhart: Dann werden wir zwei unterschiedliche Antworten auf die Frage nach dem gegenwärtigen Status des Faches und den Möglichkeiten einer Weiterentwicklung geben müssen. In der Diagnose sind wir uns ja einig: Die gegenwärtige Geographie kann recht anschaulich durch die Bildmetapher eines Donuts gekennzeichnet werden. In der Mitte ist ein Loch, es gibt kein Zentrum. Mein Vorschlag lautet: Es ist sinnvoll, für das Fach eine neue Mitte zu schaffen. Dazu plädiere ich für die ausdrückliche Betonung geographischer Fundamentalien und die Orientierung an einer Universalethik wie Gerechtigkeit und Freiheit. Die Fundamentalien sind als abstrakte Perspektiven zu sehen, die von jedem Paradigma oder Ansatz aus in Wert gesetzt werden können. Ein „disziplinärer Imperialismus“ kann von ihnen also nicht ausgehen, und sie bieten vielfältige Anknüpfungspunkte für disziplinübergreifende Arbeit. Die Suche nach einem Zentrum bedeutet natürlich
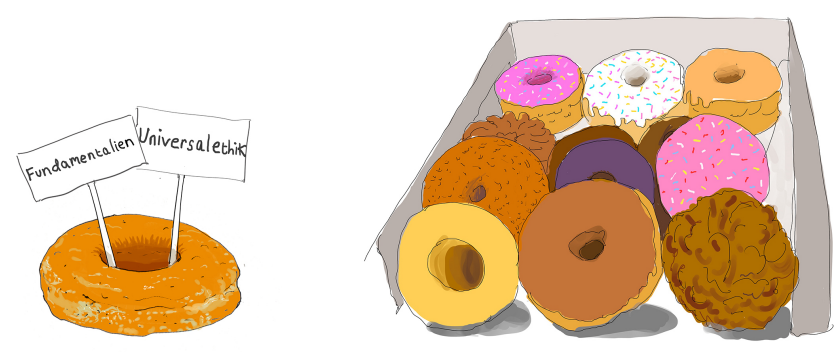

Abb. 16. Ein neuer Kern oder dezentrierende Konnektivität für eine Geographie der Zukunft? () Tom Reed Studio.

nicht, die Peripherie aufgeben zu wollen. Auch mit einem Zentrum muss die Stärke und Innovationskraft der Peripherie aufrechterhalten bleiben.

Dein Argument dagegen lautet: Wir brauchen keine neue Mitte, sie könnte ein gefährliches Disziplinierungsinstrument sein. Erkennen wir stattdessen an, dass die theoretische Innovationskraft der Disziplin häufig von den disziplinären und auch gesellschaftlichen Randzonen ausgeht. Die Öffnung des Faches gegenüber Nachbardisziplinen und Inklusion derer, die in der Disziplin am Rand stehen, sollte das Ziel sein für die Art und Weise, wie wir in Zukunft Geographie betreiben.

\section{7 ... oder dezentrierende Konnektivität?}

Carolin Schurr: Ja, genau, ich sehe gerade in der Offenheit der Geographie ihre Zukunft. Durch die ständige Pluralisierung - also durch die zentrifugalen Kräfte an den Rändern des Donuts - bleibt die Geographie innovativ und offen für neue Ansätze. Oder wie Du sagen würdest, für neue Werkzeuge im Werkzeugkoffer. Ich wünsche mir für die Zukunft eine Geographie, die als „Raum der Verbindungen“ fungiert. Dieser Raum der Verbindungen kann sich im Sinne von Deleuzes (2001) Rhizom durch Interventionen insbesondere, aber nicht nur aus den Rändern der Disziplin und interdisziplinären Saumstellen verändern. Statt darüber nachzudenken, wie wir das Loch des Donuts möglicherweise füllen könnten, finde ich es abschliessend interessanter zu konstatieren, dass es momentan viele Debatten und neue Felder in der Geographie gibt, die den Donut „Geographie“ äusserst schmackhaft und bunt machen. Momentan laufende DFG-Netzwerke zu Feministischen Geographien des neuen Materialismus, Digitalen Geographien, dem Practice Turn in der Geographie oder Räumen globaler Produktion, neue Zeitschriften wie die sub/urban oder movements, die aus dem Versuch heraus entstanden sind, eine kritische Stadt- und Migrationsforschung mit reger Beteiligung von Geograph_innen zu etablieren, oder die vielzähligen neuen Sonderforschungsbereiche, an denen die Geographie mitwirkt - all diese Forschungsinitiativen zeugen davon, dass die Geographie ein Ort der Konnektivität ist. Geographien der Zukunft sind für mich Orte der Konnektivität - Orte der 
Konnektivität, an denen inter- und transdisziplinär gedacht wird, disziplinübergreifende Sozial- und Gesellschaftstheorien aufgegriffen und für neue Fragestellungen innerhalb der Geographie ausgelotet werden. Im gegenwärtigen politischen Klima des Hassschürens gegen das Andere, des Ausschlusses, des Grenzenziehens sind für mich Inklusion der Ränder, Offenheit und Konnektivität die Basis für Geographien der Zukunft.

Datenverfügbarkeit. Für diesen Artikel wurden keine Datensätze genutzt.

Autorenmitwirkung. CS und PW haben gemeinsam die Idee und den Text entwickelt.

Interessenkonflikt. Die Autoren erklären, dass kein Interessenkonflikt besteht.

Danksagung. Wir danken Florian Dünckmann und dem Team des Geographischen Instituts der Universität Kiel für die Einladung und die Idee, den Eröffnungsvortrag zum Deutschen Kongress für Geographie in Kiel 2019 unter dem Titel „Umbrüche und Aufbrüche: Geographien der Zukunft" in Dialogform zu halten. Des Weiteren möchten wir uns bei Elisabeth Militz, Amena Schwabe, Alexander Vorbrugg und Martin Müller für kritisches Feedback, Tom Reeds Studio für die Illustrationen und Angelika Weichhart für die sorgfältige Korrektur bedanken.

Begutachtung. This paper was edited by Benedikt Korf and reviewed by one anonymous referee.

\section{Literatur}

Agnew, J. and Livingstone, D. N.: The SAGE Handbook of Geographical Knowledge, Sage, Los Angeles, 2011.

Ahmed, S.: Open Forum Imaginary Prohibitions: Some Preliminary Remarks on the Founding Gestures of the ,New Materialism', Eur. J. Women's Stud., 15, 23-39, https://doi.org/10.1177/1350506807084854, 2008.

Alaimo, S.: Bodily Natures: Science, Environment, and the Material Self, Indiana University Press, Bloomington, 2010.

Alaimo, S. and Hekman, S.: Material Feminisms, Indiana University Press, Bloomington, IN, 2008.

Barad, K.: Posthumanist Performativity: Toward an Understanding of How Matter Comes to Matter, in: Material feminisms, edited by: Alaimo, S. und Hekman, S., Indiana University Press, Bloomington, 120-154, 2008.

Barnes, T.: Retheorizing Economic Geography: From the Quantitative Revolution to the „Cultural Turn“, Ann. Assoc. Am. Geogr., 91, 546-565, 2001.
Bartels, D.: Zur wissenschaftstheoretischen Grundlegung einer Geographie des Menschen, in: Erdkundliches Wissen, FranzSteiner Verlag, Wiesbaden, 1986.

Bauriedl, S., Fleischmann, K., Strüver, A. und Wucherpfennig, C.: Verkörperte Räume, „Verräumte Körper“. Zu einem poststrukturalistischen Verständnis der Wechselbeziehungen von Körper und Raum, Geogr. Helv., 55, 130-137, 2000.

Berndt, C. und Boeckler, M.: Kulturelle Geographien der Ökonomie: Zur Performativität von Märkten, in: Kulturelle Geographien, Herausgeber: Berndt, C. und Pütz, R., transcript, Bielefeld, 213-258, 2008

Black, D.: Where Bodies End and Artefacts Begin Tools, Machines and Interfaces, Body Soc., 20, 31-60, https://doi.org/10.1177/1357034x13506946, 2014.

Bonds, A.: Racing Economic Geography: The Place of Race in Economic Geography, Geogr. Compass, 7, 398-411, https://doi.org/10.1111/gec3.12049, 2013.

Borsdorf, A.: Länderkunde abgeschafft? Zur Bedeutung der Regionalgeographie im deutschen Sprachraum vor und 50 Jahre nach Kiel unter besonderer Berücksichtigung der geographischen Lateinamerikanistik. Festvortrag zum 80. Geburtstag von Christoph Stadel, Mitt. Österr. Geogr. Gesell., 1, 351-359, https://doi.org/10.1553/moegg160s351, 2019.

Braun, B.: Book Review Forum: Vibrant Matter: A Political Ecology of Things, Dialog. Human Geogr., 1, 390-393, https://doi.org/10.1177/2043820611421562, 2011.

Brenner, N. and Schmid, C.: Planetary Urbanisation, in: Urban constellations, edited by: Gandy, M., Jovis, Berlin, 10-13, 2011.

Brenner, N. and Schmid, C.: Towards a New Epistemology of the Urban?, City, 19, 151-182, https://doi.org/10.1080/13604813.2015.1014712, 2015.

Brown, M.: Gender and Sexuality I: Intersectional Anxieties, Prog. Human Geogr., 36, 541-550, https://doi.org/10.1177/0309132511420973, 2012.

Castree, N.: Economy and Culture are Dead! Long Live Economy and Culture!, Prog. Human Geogr., 28, 204-226, https://doi.org/10.1191/0309132504ph468oa, 2004.

Colls, R. und Evans, B.: Making Space for Fat Bodies: A critical Account of ,the Obesogenic Environment ', Prog. Human Geogr., 36, 733-753, https://doi.org/10.1177/0309132513500373, 2013.

Crenshaw, K.: Demarginalizing the Intersection of Race and Sex: A Black Feminist Critique of Antidiscrimination Doctrine, Univers. Chicago Legal Forum, 1, 139-167, 1989.

Curry, L.: Quantitative Géographie, The Canadian Geographer/Le Géographe canadien, 11, 265-279, https://doi.org/10.1111/j.1541-0064.1967.tb00472.x, 1967.

Deleuze, G.: „Rhizom“. Short Cuts, Zweitausendeins, Frankfurt am Main, 2001

Elias, N.: What is Sociology? European Perspectives: A Series in Social Thought and Cultural Criticism, Columbia University Press, New York, 1978.

Fischer, W. L.: Mathematik und Ethnomathematik: Globalität und Globalisierung in der Geschichte der Mathematik, in: Forschungsgesellschaft: Fremdheit im Prozess der Globalisierung. Bereicherung? Bedrohung? Nivellierung?, Schriftenreihe der Forschungsgesellschaft Wilhelminenberg, LIT, Münster, 162-177, 2006. 
Franklin, S.: The Cyborg Embryo: Our Path to Transbiology, Theor. Cult. Soc., 23, 167-187, https://doi.org/10.1177/0263276406069230, 2006.

Gebhardt, H.: „Jetzt wächst zusammen, was zusammengehört?““ Diskussionsbemerkungen zu den Vorträgen auf dem Münchner Symposium zur Zukunft der Geographie, in: Eine Dokumentation, Münchener Geographische Hefte 85, Integrative Ansätze in der Geographie - Vorbild oder Trugbild?, Herausgeber: Heinritz, G., Münchner Symposium zur Zukunft der Geographie, 28. April 2003, München, 65-72, 2003.

Gibson-Graham, J. K.: Rethinking the Economy with Thick Description and Weak Theory, Curr. Anthropol., 55, 147-153, 2014.

Giudice, C. and Giubilaro, C.: Re-Imagining the Border: Border Art as a Space of Critical Imagination and Creative Resistance, Geopolitics, 20, 79-94, https://doi.org/10.1080/14650045.2014.896791, 2015.

Haraway, D.: Situated Knowledges: The Science Question in Feminism and the Privilege of Partial Perspective, Feminist Stud., 14, 575-599, 1988.

Haraway, D.: Simians, Cyborgs, and Women: The Reinvention of Nature, Free Association Books, London, 1991.

Haraway, D.: When Species Meet, University of Minnesota Press, Minneapolis, 2008.

Hard, G.: Die „Landschaft“ der Sprache und die „Landschaft“ der Geographen. Semantische und forschungslogische Studien zu einigen zentralen Denkfiguren in der deutschen geographischen Literatur, Colloquim Geographicum, Bonn, 1970.

Hodgetts, T. and Lorimer, J.: Methodologies for Animals' Geographies: Cultures, Communication and Genomics, Cult. Geogr., 22, 285-295, 2015.

Hooks, B.: Choosing the Margin as a Space of Radical Openness, Framework: J. Cinema Media, 36, 15-23, 1989.

Hooks, B.: Feminist Theory: From Margin to Center, Pluto Press, London, 2000.

Hopkins, P.: Social Geography I: Intersectionality, Prog. Human Geogr., 36, 441-450, https://doi.org/10.1177/0309132517743677, 2017.

Jessop, B. and Oosterlynck, S.: Cultural Political Economy: On Making the Cultural Turn without Falling into Soft Economic Sociology, Geoforum, 39, 1155-1169, https://doi.org/10.1016/j.geoforum.2006.12.008, 2008.

Koopman, S.: Imperialism Within: Can the Master's Tools Bring down Empire?, ACME - Int. J. Crit. Geogr., 7, 283-307, 2008.

Kramer, C.: „Alles hat seine Zeit“ - die „Time Geography“ im Lichte des „Material Turn“, in: Anthologie zur Sozialgeographie, Abhandlungen zur Geogaphie und Regionalforschung, Herausgeber: Weixelbaumer, H., Institut für Geographie und Regionalforschung der Universität Wien, Wien, 83-105, 2012.

Latour, B.: We Have Never Been Modern, Harvard University Press, Cambridge, MA, 1993.

Latour, B.: Body, Cyborgs and the Politics of Incarnation, in: The Body, Herausgeber: Sweeney, S. T. and Hodder, I., Cambridge University Press, Cambridge, New York, 127-142, 2002.

Law, J.: After Method: Mess in Social Science Research, Routledge, London, 2004.

Longhurst, R.: Bodies: Exploring Fluid Boundaries, Routledge, London, 2001.
Longhurst, R. and Johnston, L.: Bodies, Gender, Place and Culture: 21 Years on, Gender Place Cult., 21, 267-278, https://doi.org/10.1080/0966369X.2014.897220, 2014.

Lorde, A.: Sister Outsider: Essays and Speeches, The Crossing Press, Freedom, CA, 1984.

Marquardt, N. und Strüver, A.: Körper. Machtgeladene Intraaktionen zwischen Biologischem und Sozialem, in: Raumproduktionen II. Theoretische Kontroversen und politische Auseinandersetzungen, Herausgeber: Vogelpohl, A., Michel, B., Lebuhn, H., Hoerning, J. und Belina, B., Westfälisches Dampfboot, Münster, 38-59, 2018.

McDowell, L.: Working Bodies: Interactive Service Employment and Workplace Identities, Wiley-Blackwell, Oxford, 2009.

McDowell, L. and Court, G.: Performing Work: Bodily Representations in Merchant Banks, Environ. Plan. D, 12, 727-750, 1994.

McKittrick, K.: On Plantations, Prisons, and a Black Sense of Place, Social Cult. Geogr., 12, 947-963, https://doi.org/10.1080/14649365.2011.624280, 2011.

McLean, H.: In Praise of Chaotic Research Pathways: A Feminist Response to Planetary Urbanization, Environ. Plan. D, 36, 547555, https://doi.org/10.1177/0263775817713751, 2018.

Mignolo, W. D.: Local Histories/Global Design: Coloniality, Subaltern Knowledges, and Border Thinking, Princeton University Press, Princeton, New Jersey, 2000.

Mignolo, W. D.: Capitalismo y geopolítica del conocimiento: el eurocentrismo y la filosofía de la liberación en el debate intelectual contemporáneo, Ediciones del Signo, Buenos Aires, Argentina, 2001.

Mollett, S. and Faria, C.: The Spatialities of Intersectional Thinking: Fashioning Feminist Geographic Futures, Gender Place Cult., 25, 565-577, https://doi.org/10.1080/0966369X.2018.1454404, 2018.

Monk, J. and Hanson, S.: On Not Excluding Half of the Human in Human Geography, Profess. Geogr., 34, 11-23, 1982.

Müller, M.: In Search of the Global East: Thinking between North and South, Geopolitics, https://doi.org/10.2139/ssrn.2881296, in press, 2019.

Müller, M. and Schurr, C.: Assemblage Thinking and Actor-Network Theory: Conjunctions, Disjunctions, Cross-Fertilisations, T. Inst. Brit. Geogr., 41, 217-229, https://doi.org/10.1111/tran.12117, 2016.

Nelson, L.: Bodies (and Spaces) Do Matter: The Limits of Performativity, Gender Place Cult., 6, 331-353, 1999.

Nelson, L.: Geographies of State Power, Protest, and Women's Political Identity Formation in Michoacán, Mexico, Ann. Assoc. Am. Geogr., 96, 366-389, https://doi.org/10.1111/j.14678306.2006.00482.x, 2006.

Oberhauser, A. M.: Feminism and Economic Geography: Gendering Work and Working Gender, in: A Companion to Economic Geography, edited by: Barnes, T. J. and Sheppard, E. S., Blackwell, Malden, 60-76, 2000.

Oswin, N.: Planetary Urbanization: A View from Outside, Environ. Plan. D, 36, 540-546, https://doi.org/10.1177/0263775816675963, 2018.

Oswin, N.: Enough, in: Keywords in Radical Geography: Antipode at 50, edited by: Antipode Editorial Collective, John Wiley and Sons Inc., Hoboken, NJ, 113-117, 2019.

Peake, L., Patrick, D., Reddy, R. N., Sarp Tanyildiz, G., Ruddick, S., and Tchoukaleyska, R.: Placing Planetary Urbanization 
in other Fields of Vision, Environ. Plan. D, 36, 374-386, https://doi.org/10.1177/0263775818775198, 2018.

Peck, J.: Economic Geography: Island Life, Dialog. Human Geogr., 2, 113-133, 2012.

Peck, J. and Olds, K.: Report: The Summer Institute in Economic Geography, Econ. Geogr., 83, 309-318, https://doi.org/10.1111/j.1944-8287.2007.tb00356.x, 2007.

Pickles, J.: The Cultural Turn and the Conjunctural Economy: Economic Geography, Anthropology, and Cultural Studies, in: The Wiley-Blackwell Companion to Economic Geography, edited by: Barnes, T., Peck, J., and Sheppard, E., John Wiley \& Sons, Ltd, Chichester, 537-551, 2012.

Plessner, H.: Die verspätete Nation. Über die politische Verführbarkeit bürgerlichen Geistes, Kohlhammer Verlag, Stuttgart, 1959 [1935].

Pollard, J., McEwan, C., Laurie, N., and Stenning, A.: Economic Geography Under Postcolonial Scrutiny, T. Inst. Brit. Geogr., 34, 137-142, https://doi.org/10.1111/j.1475-5661.2009.00336.x, 2009.

Quijano, A.: Coloniality of Power and Eurocentrism in Latin America, Int. Sociol., 15, 217-234, 2000.

Reddy, R. N.: The Urban Under Erasure: Towards a Postcolonial Critique of Planetary Urbanization, Environ. Plan. D, 36, 529539, https://doi.org/10.1177/0263775817744220, 2018.

Ruddick, S., Peake, L., Tanyildiz, G. S., and Patrick, D.: Planetary Urbanization: An Urban Theory for Our Time?, Environ. Plan. D, 36, 387-404, https://doi.org/10.1177/0263775817721489, 2018.

Schatzki, T.: The Site of the Social. A Philosophical Account of the Constitution of Social Life and Change, Penn State University Press, Philadelphia, 2002.

Schneider, H.: Nachhaltigkeit als regulative Idee in der geographischen Stadt- und Tourismusforschung, Geographie, Forschung und Wissenschaft Hamburg, 2006.

Schurr, C.: From Biopolitics to Bioeconomies: The ART of (Re-)Producing White Futures in Mexico's Surrogacy Market, Environ. Plan. D, 35, 241-262, 2017.

Schurr, C., Müller, M., and Imhof, N.: Who Makes Geographical Knowledge? The Gender of Geography's Gatekeepers, Profess. Geogr., in press, 2020.

Sedlacek, P. (Hrsg.): Einleitung, in: Zur Situation der deutschen Geographie zehn Jahre nach Kiel, Selbstverlag des Fachbereichs 2 der Universität Osnabrück, Osnabrück, 7-9, 1979.

Steinbrink, M., Aufenvenne, P. und Schmidt, J.-B.: Poster: „Geographie - Ein Mythos auf dem empirischen Prüfstand“, online aufrufbar: https://www.academia.edu/28642086/Poster_ Geographie_Ein_Mythos_auf_dem_empirischen_Prüfstand_ presented_at_University_of_Osnabrueck_2014 (letzter Zugriff: 8. Dezember 2019), Osnabrueck, 2014.

Storper, M. and Scott, A. J.: Current Debates in Urban Theory: A Critical Assessment, Urban Stud., 53, 1114-1136, https://doi.org/10.1177/0042098016634002, 2016.
Strüver, A.: KörperMachtRaum und RaumMachtKörper: Bedeutungsverflechtungen von Körpern und Räumen, in: Geschlechterverhältnisse, Raumstrukturen, Ortsbeziehungen: Erkundungen von Vielfalt und Differenz im spatial turn, Reihe, Herausgeber: Bauriedl, S., Schier, M. und Strüver, A., Forum Frauen- und Geschlechterforschung, Münster, 217-238, 2010.

Strüver, A.: Zum Zusammenwirken von Materialität und Repräsentation: Donna Haraway and Judith Butler, in: Theorien in der Raum- und Stadtforschung, Herausgeber: Oßenbrügge, J. und Vogelpohl, A., Westfälisches Dampfboot, Münster, 290-309, 2014.

Strüver, A.: Von der Inkorporierung und Verkörperung des Sozialen zur Somatisierung der Umwelt: Posthumanistische Überlegungen zum biosozialen Subjekt, Geogr. Helv., 74, 223-233, https://doi.org/10.5194/gh-74-223-2019, 2019.

Thrift, N.: Non-Representational Theory: Space, Politics, Affect, Routledge, London, 2008.

Thrift, N. and Olds, K.: Refiguring the Economic in Economic Geography, Prog. Human Geogr., 20, 311-337, https://doi.org/10.1177/030913259602000302, 1996.

Tolia-Kelly, D. P.: The Geographies of Cultural Geography III: Material Geographies, Vibrant Matters and Risking Surface Geographies, Prog. Human Geogr., 37, 153-160, https://doi.org/10.1177/0309132512439154, 2013.

Valentine, G.: Theorizing and Researching Intersectionality: A Challenge for Feminist Geography, Profess. Geogr., 59, 10-21, 2007.

Weichhart, P.: Geography as a ,Multi-Paradigm Game“ - a Pluralistic Discipline in a Pluralistic Post-Industrial Society, in: Proceedings of an International Conference held at Vechta, 8-12 September 1986, The Role of Geography in a Post-Industrial Society, Herausgeber: Windhorst, H.-W., Vechtaer Arbeiten zur Geographie und Regionalwissenschaft, Vechta, 49-54, 1987.

Weichhart, P.: Physische Geographie und Humangeographie eine schwierige Beziehung: Skeptische Anmerkungen zu einer Grundfrage der Geographie und zum Münchner Projekt einer „Integrativen Umweltwissenschaft“, in: „Integrative Ansätze in der Geographie - Vorbild oder Trugbild?“, Münchner Symposium zur Zukunft der Geographie, 28. April 2003, Eine Dokumentation, Münchener Geographische Hefte 85, Herausgeber: Heinritz, G., München, 17-34, 2003.

Weichhart, P.: Entwicklungslinien der Sozialgeographie. Von Hans Bobek bis Benno Werlen, Sozialgeographie kompakt, Franz-Steiner Verlag, Wiesbaden, 2019 [2008].

Werlen, B.: Kiel 1969 - Leuchtturm oder Irrlicht?, Geogr. Helv., 69, 293-299, https://doi.org/10.5194/gh-69-293-2014, 2014. 